\title{
ESTUDO ELETROQUÍMICO DO AZUL DE METILENO ADSORVIDO SOBRE SÍLICA GEL QUIMICAMENTE MODIFICADA COM ÓXIDO DE NIÓBIO
}

\author{
Débora de Almeida Schiavo, Elizabeth Fátima Perez, Lauro Tatsuo Kubota"
}

Instituto de Química - Unicamp - 13083-970 - Campinas-SP

Recebido em 20/8/99; aceito em 17/3/00

\begin{abstract}
ELECTROCHEMICAL STUDY OF METHYLENE BLUE ADSORBED ON THE SILICA GEL MODIFIED WITH NIOBIUM OXIDE. Methylene blue (AM) was immobilised on surface of the silica gel modified with niobium oxide. This material was incorporated in a carbon paste electrode, which showed a redox couple in a potential of $E=-113 \mathrm{mV}$ vs SCE in KCl solution at $\mathrm{pH}$ 7.0. The formal potential, in $0.5 \mathrm{~mol} \mathrm{~L}^{-1} \mathrm{KCl}$ at $\mathrm{pH} 7.0$, shifted about $290 \mathrm{mV}$ towards more positive values compared to those observed for AM solubilized in aqueous solution. The dependence on the formal potential with solution pH between 2 and 7 was much lower than those observed for AM solubilized in aqueous solution.
\end{abstract}

Keywords: methylene blue; silica gel modified with niobium oxide; carbon paste electrode.

\section{INTRODUÇÃO}

O azul de metileno ${ }^{1,2}$, é um corante da classe das fenotiazinas ${ }^{3}$ e tem despertado interesse devido a sua propriedade eletrocatalítica frente ao $\mathrm{NADH}^{4}$, que é a coenzima das enzimas dehidrogenases, e por sua semelhança com as flavinas, as quais são importantes agentes redox biológicos participando de várias reações enzimáticas. O mecanismo de reação redox das flavinas ${ }^{5,6}$ nos processos catalíticos ainda é pouco compreendido, dessa maneira um estudo mais aprofundado com corantes semelhantes as flavinas, pode ajudar a desvendar esse mecanismo.

Os eletrodos quimicamente modificados (EQM) têm sido muito utilizados para diminuir o sobrepotencial que alguns analitos requerem para se oxidar ou reduzir, na tentativa de minimizar os problemas de interferentes e estabilidade dos sensores durante as análises ${ }^{7}$. Dentre os EQM's destacam-se os eletrodos de pasta de carbono ${ }^{8}$, pois permitem a incorporação de matrizes inorgânicas (sílicas ${ }^{9}$, zeólitas $^{10}, \operatorname{argilas}^{11}$ ), as quais conferem características específicas ao eletrodo ${ }^{12}$. Os eletrodos de pasta de carbono ainda apresentam como vantagens baixa corrente de fundo, superfície facilmente renovada, facilidade de preparação e ampla faixa de potencial de trabalho ${ }^{7}$.

A sílica gel quimicamente modificada ${ }^{13,14}$ tem recebido especial atenção na imobilização de mediadores hidrossolúveis, impedindo a lixiviação do mesmo, conferindo maior estabilidade ao eletrodo. Entre suas propriedades destaca-se sua alta área superficial, porosidade controlada, resistência mecânica e estabilidade química ${ }^{15}$, as quais permitem que uma maior quantidade de mediador seja incorporado no eletrodo.

Neste trabalho será descrito o comportamento eletroquímico do azul de metileno, imobilizado na sílica modificada com óxido de nióbio, incorporado em eletrodo de pasta de carbono e dessa forma estudar como o mediador interage com a matriz, o que confere características distintas ao mesmo.

\section{PARTE EXPERIMENTAL}

\section{Síntese da sílica modificada com óxido de nióbio $(\mathrm{SN})^{16}$}

A sílica gel modificada foi preparada a partir de $40 \mathrm{~g}$ de silica gel 60 (Fluka), ativada por sete horas em estufa a $120^{\circ} \mathrm{C}, 22 \mathrm{~g}$ de pentacloreto de nióbio (Aldrich) dissolvido em etanol absoluto (Merck), e $200 \mathrm{~mL}$ de tetracloreto de carbono (Merck).

A reação processou-se por oito horas sob refluxo em banhomaria. Decorrido este tempo a sílica foi filtrada, lavada e seca à vácuo. À seguir a mesma foi hidrolisada por imersão em água destilada, agitando-se por quinze minutos, sendo posteriormente seca em estufa a $90^{\circ} \mathrm{C}$.

O teor de nióbio incorporado na superfície da sílica gel foi obtido através de fuorescência de raios-X utilizando-se o pentacloreto de nióbio como padrão e o método de parâmetros fundamentais no espectrômetro Spectrace 5000.

\section{Adsorção do corante na SN}

O azul de metileno (AM) foi adsorvido na SN pela imersão de $200 \mathrm{mg}$ de $\mathrm{SN}$ em $5 \mathrm{~mL}$ de solução $0,1 \%(\mathrm{~m} / \mathrm{V})$ do corante durante uma hora. A seguir o material SNAM foi filtrado, lavado com água deionizada, até o sobrenadante ficar incolor, e deixado secar por uma semana à temperatura ambiente.

O teor de AM no material SNAM foi obtido por análise elementar utilizando-se o aparelho Elementar Analyser PE2400. O espectro UV-VIS do mediador adsorvido na superfície da sílica foi obtido no espectrofotômetro HP-8452A.

\section{Medidas eletroquímicas}

As medidas eletroquímicas foram realizadas no potenciostato EG\&G da Princeton Applied Research modelo 273A, conectado a um microcomputador tipo PC para controle de potencial e aquisição dos dados, utilizando-se eletrodo de calomelano saturado (ECS) como referência e um fio de platina como auxiliar. O eletrodo de trabalho foi constituído de um eletrodo de pasta de carbono, cuja composição é 1:1 de grafite e SNAM, e gotas de óleo mineral foram adicionadas como aglutinante.

\section{Estudo eletroquímico}

Os voltamogramas cíclicos foram obtidos em diferentes eletrólitos suportes à $0,5 \mathrm{~mol} \mathrm{~L}^{-1} \mathrm{e}$ velocidades de varredura. Estudou-se o efeito da concentração e do pH (2 à 7) do eletrólito suporte, bem como a estabilidade do eletrodo.

Todas a medidas foram realizadas em atmosfera de nitrogênio e os reagentes utilizados nos experimentos foram de grau analítico. 


\section{RESULTADOS E DISCUSSÃO}

\section{Obtenção e caracterização da matriz SN}

A sílica tem sua superfície modificada segundo as reações abaixo $^{16}$ :

$$
\begin{gathered}
\mathrm{NbCl}_{5}+5 \mathrm{C}_{2} \mathrm{H}_{5} \mathrm{OH} \longrightarrow \mathrm{Nb}\left(\mathrm{OC}_{2} \mathrm{H}_{5}\right)_{5}+5 \mathrm{HCl} \\
n-\mathrm{SiOH}+\mathrm{Nb}\left(\mathrm{OC}_{2} \mathrm{H}_{5}\right)_{5} \longrightarrow \mathrm{C}_{2} \mathrm{H}_{5} \mathrm{OH} \\
(-\mathrm{SiO})_{\mathrm{n}} \mathrm{Nb}\left(\mathrm{OC}_{2} \mathrm{H}_{5}\right)_{5-n}+ \\
(-\mathrm{SiO})_{n} \mathrm{Nb}\left(\mathrm{OC}_{2} \mathrm{H}_{5}\right)_{5-n}+(5-n) \mathrm{H}_{2} \mathrm{O} \longrightarrow(-\mathrm{SiO})_{n} \mathrm{Nb}(\mathrm{OH})_{5-n} \\
+(5-n) \mathrm{C}_{2} \mathrm{H}_{5} \mathrm{OH}
\end{gathered}
$$

Os sítios $\mathrm{Nb}(\mathrm{OH})_{5-n}$ são os responsáveis pela adsorção do mediador na superfície da sílica.

$$
\mathrm{SN}^{-} \mathrm{H}^{+}+\mathrm{AM}^{+} \mathrm{Cl}^{-} \varnothing \mathrm{SN}^{-} \mathrm{AM}^{+}+\mathrm{HCl}
$$

Através da análise da sílica por fluorescência de raio-X obteve-se que a quantidade de nióbio na superfície da mesma é de $0,26 \mathrm{mmol} \mathrm{de} \mathrm{Nb} / \mathrm{g}$ de material. A análise de área superficial BET revelou uma grande superfície do material $\left(375 \mathrm{~m}^{2}\right.$ $\mathrm{g}^{-1}$ ), esta constitui uma das maiores vantagens na utilização da sílica como matriz inerte, pois permite que uma maior quantidade de mediador possa ser adsorvida na matriz. A análise elementar revelou que a $\mathrm{SN}$ adsorveu $41 \mu \mathrm{mol}$ de $\mathrm{AM} / \mathrm{g}$ de $\mathrm{SN}$, quantidade essa comparável com outros compostos adsorvidos descritos na literatura ${ }^{17}$.

Através do espectro UV-VIS de SNAM, Figura 1, nota-se que o corante mantém as mesmas características tanto imobilizado como solúvel, não havendo alteração dos máximos dos picos, nem da razão entre eles.

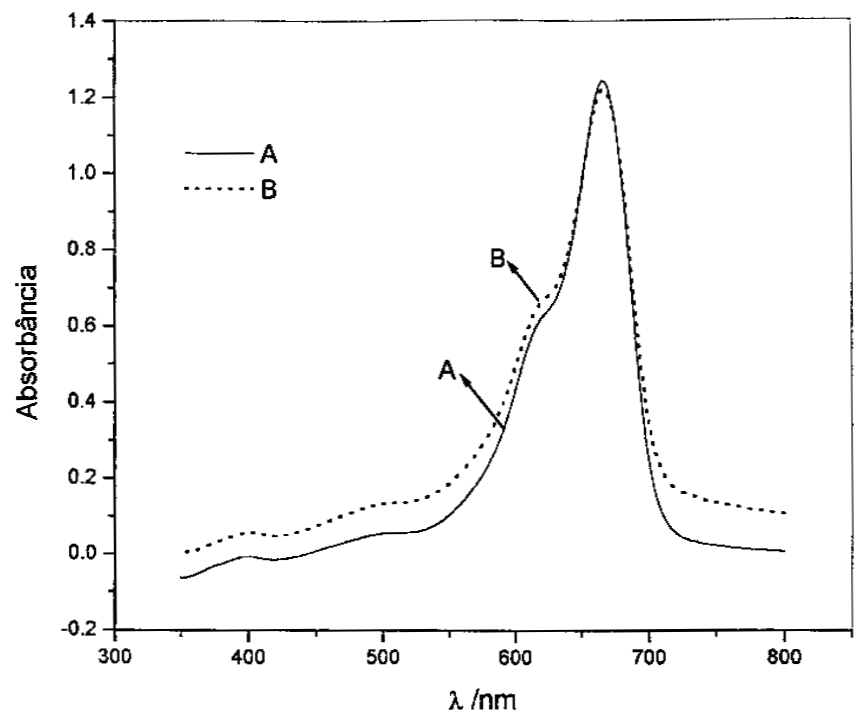

Figura 1. Espectro de absorbância UV-VIS do azul de metileno, A) solúvel, B) adsorvido na matriz.

\section{Propriedades eletroquímicas do AM adsorvido em SN}

A imobilização do AM na matriz sílica gel quimicamente modificada com óxido de nióbio, permitiu a construção do eletrodo de pasta de carbono, cuja resposta ciclovoltamétrica pode ser visualizada na Figura 2.

$\mathrm{O}$ potencial médio $\left(\mathrm{E}_{\mathrm{m}}\right)$ obtido no estudo ciclovoltamétrico do AM, mostrou que entre os ânions estudados, apenas o íon acetato apresenta um comportamento diferente, Tabela 1, revelando uma maior interação deste com o mediador. Esse fato pode ser explicado pela grande afinidade do nióbio pelo grupo carboxila, dessa forma facilitando a interação do acetato com o mediador, aumentando a densidade eletrônica estabilizando a forma oxidada do AM. Nesta mesma Tabela, pode-se verificar que dentre os diferentes cátions estudados, apenas o íon $\mathrm{NH}_{4}{ }^{+}$ apresentou o $\mathrm{E}_{\mathrm{m}}$ deslocado para valores mais negativos, isto provavelmente deve-se ao fato do $\mathrm{NH}_{4}{ }^{+}$estabilizar o estado oxidado do AM, provavelmente devido a uma interação do $\mathrm{NH}_{4}{ }^{+}$ com a matriz, enfraquecendo o efeito da mesma sobre o AM.

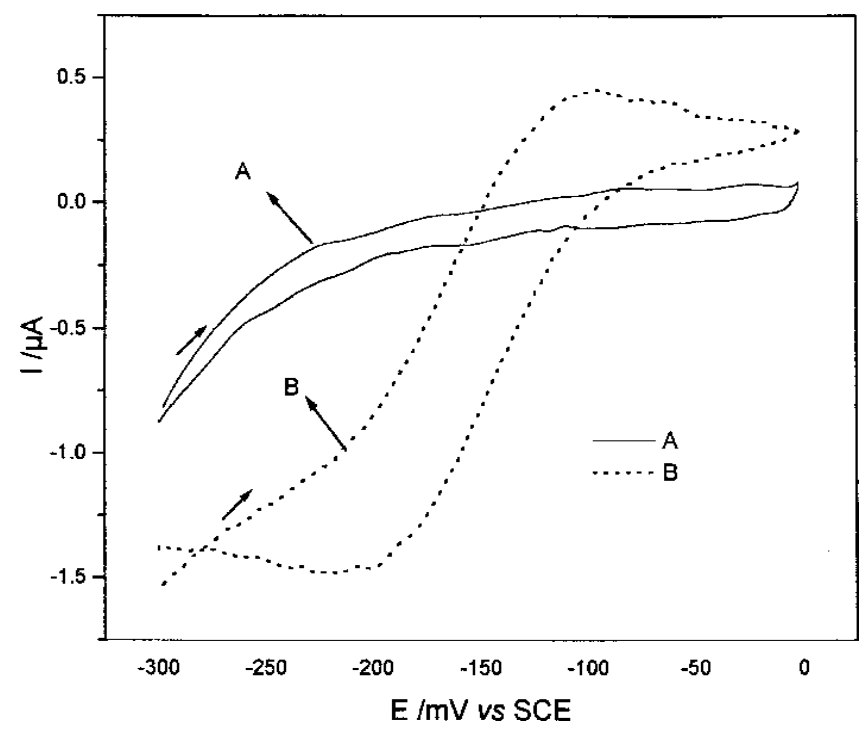

Figura 2. Voltamogramas do eletrodo de pasta de carbono em $\mathrm{KCl}$ $0,5 \mathrm{~mol} \mathrm{~L}^{-1} \mathrm{pH}=7, v=10 \mathrm{mV} \mathrm{s}^{-1}$. A) apenas a sílica modificada, B) sílica adsorvida com AM.

Tabela 1. Potenciais médios, $\mathrm{E}_{\mathrm{m}}$, obtidos para os diferentes ânions e cátions $\mathrm{v}=10 \mathrm{mV} \mathrm{s}^{-1}$, soluções $0,5 \mathrm{~mol} \mathrm{~L}^{-1} \mathrm{pH} \sim 7$

\begin{tabular}{cccc}
\hline$\hat{\mathrm{A} n i o n}$ & $\mathrm{E} / \mathrm{mV}$ & $\mathrm{Cátions}$ & $\mathrm{E} / \mathrm{mV}$ \\
\hline $\mathrm{NO}_{3}^{-}$ & -166 & $\mathrm{Li}^{+}$ & -144 \\
$\mathrm{Cl}^{-}$ & -157 & $\mathrm{~K}^{+}$ & -133 \\
$\mathrm{SO}_{4}{ }^{2-}$ & -154 & $\mathrm{NH}_{4}^{+}$ & -179 \\
$\mathrm{Ac}^{-}$ & -197 & $\mathrm{Ca}^{2+}$ & -132 \\
$\mathrm{ClO}_{4}^{-}$ & -166 & $\mathrm{Na}^{+}$ & -157 \\
\hline
\end{tabular}

O eletrodo modificado apresentou uma relação linear entre corrente e velocidade de varredura, mostrando que a espécie eletroativa está fortemente adsorvida na superfície da matriz.

A variação do $\mathrm{pH}$ da solução de eletrólito suporte não afetou significativamente o potencial médio do eletrodo até $\mathrm{pH} 4$, Figura 3, abaixo deste, ocorre um grande deslocamento para valores de potencial mais positivos. Quando o mediador encontra-se em solução, observa-se que o potencial é altamente afetado pelo $\mathrm{pH}$, o qual foi calculado a partir da equação $(1)^{18}$. Este fato revela o caráter ácido da matriz, permitindo que o processo seja independente do $\mathrm{pH}$ do meio até $\mathrm{pH} 4$, abaixo deste valor o efeito da matriz é minimizado, sugerindo que a proteção da mesma não é suficiente para impedir a participação do próton no processo redox.

$\mathrm{E}_{\mathrm{m}}=0,532+\ln \left(\left[\mathrm{H}^{+}\right]^{3}+\left(3,0210^{-5}\right)\left[\mathrm{H}^{+}\right]^{2}+\right.$ $\left.\left(3,0210^{-5}\right)\left(1,4110^{-6}\right)\left[\mathrm{H}^{+}\right]\right)$

A concentração do eletrólito suporte também não afeta significativamente na resposta do eletrodo, Figura 4. O eletrodo após vários ciclos (30 ciclos) não apresentou queda significativa da corrente, mostrando que o material esta fortemente adsorvido na superfície da sílica, apresentando uma boa estabilidade. 


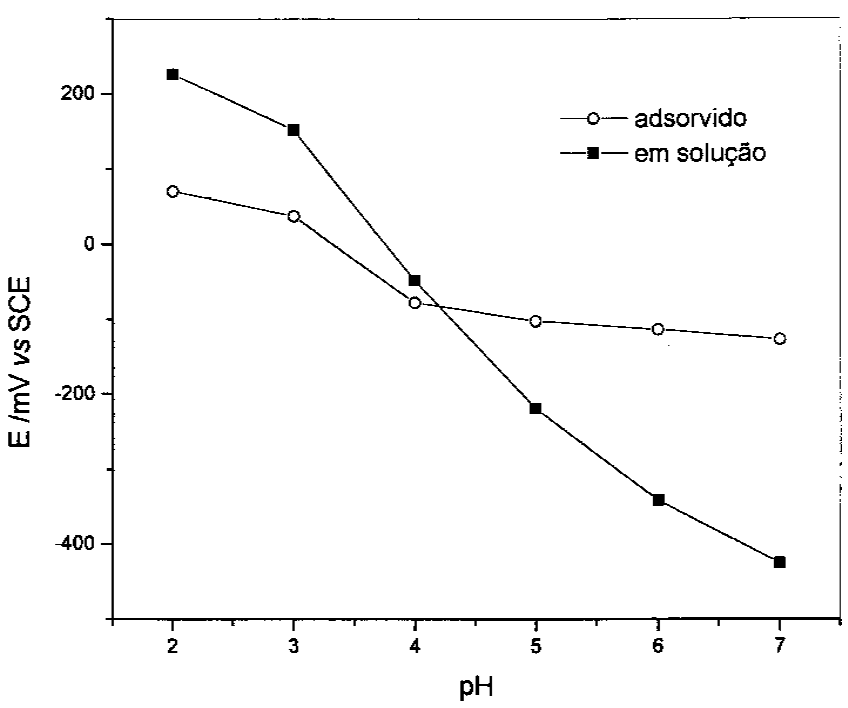

Figura 3. Potenciais médios obtidos para eletrodo de pasta de carbono em função do pH. (A) imobilizado na matriz, (B) solúvel.

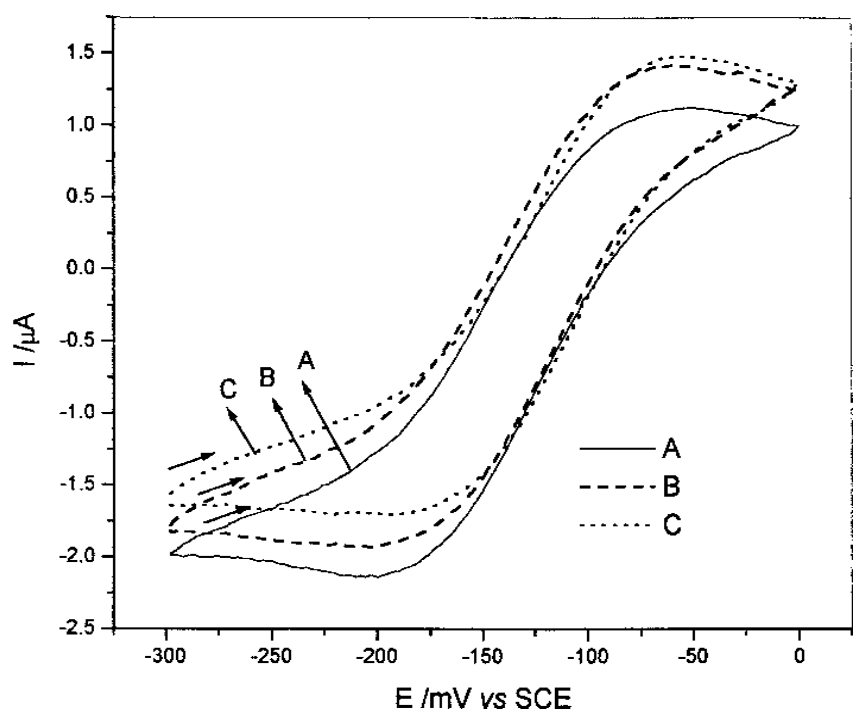

Figura 4. Voltamogramas do eletrodo de pasta de carbono em $\mathrm{KCl}$ $p H=7, v=10 \mathrm{mV} \mathrm{s}^{-1}$. A) $0,1 \mathrm{~mol} \mathrm{~L} L^{-1}$, B) $0,5 \mathrm{~mol} \mathrm{~L}^{-1}$, C) $1,0 \mathrm{~mol} \mathrm{~L}$.

O azul de metileno apresenta propriedades eletrocatalíticas frente ao NADH, quando imobilizado em fosfato de zircônio ${ }^{19}$. Dessa maneira testou-se o uso desse eletrodo para essa finalidade, ao contrário do observado na literatura, o processo eletrocatalítico não ocorre, não há um aumento significativo da corrente anódica. Esse fato sugere que a ligação do mediador com o suporte SN deve ocorrer pelo sítio ativo do AM, onde ocorre a transferência de $\mathrm{H}^{+}$, impedindo o processo eletrocatalítico, este fato vem a mostrar a dependência do $\mathrm{H}^{+}$no processo redox do NADH.

\section{CONCLUSÕES}

A matriz de sílica gel quimicamente modificada com óxido de nióbio mostrou-se um bom suporte para o azul de metileno, revelando uma grande afinidade pelo mediador, o que conferiu ao eletrodo de pasta de carbono grande estabilidade.

$\mathrm{O}$ eletrodo de pasta de carbono apresentou potencial médio em valores mais positivos do que o observado para o mediador solúvel, o que revela o caráter ácido da matriz. Essa característica também permite que até $\mathrm{pH} 4$, o potencial seja independente da acidez do meio.

O estudo da eletrocatálise revelou que a interação entre o azul de metileno ocorre no sítio ativo onde ocorre a transferência de próton entre o NADH e mediador, o que impediu o processo eletrocatalítico.

\section{AGRADECIMENTOS}

Ao CNPq, FAPESP e PRONEX pelo suporte financeiro ao projeto.

\section{REFERÊNCIAS}

1. Leon, L. E.; Talanta 1996, 43, 1275.

2. Leventis, N.; Chen, M. G.; Chem. Mater 1997, 9, 2621

3. Ju, H.; Zhou, J.; Cai, C.; Chen, H.; Electroanalysis 1995 , 7, 1165 .

4. Persson, B.; J. Electroanal. Chem. 1990, 287, 61.

5. Niemz, A.; Imbriglio, J.; Rotello, V. M.; J. Am. Chem. Soc. 1997, 119, 887.

6. Bruice, T. C.; J. Am. Chem. Soc. 1980, 13, 256.

7. Mafatle, T. J.; Nyokong, T.; J. Electroanal. Chem. 1996, 408, 213.

8. Kalcher, K.; Kauffmann, J.-M.; Wang, J.; Svancara, I.; Vytras, K.; Neuhold, C.; Yang, Z.; Electroanalysis 1995, 7, 5 .

9. Rosatto, S. S.; Kubota, L. T.; Oliveira Neto, G.; Anal Chim. Acta 1999, 390, 65.

10. Rolison, D. R.; Chem. Rev. 1990, 90, 867.

11. Sosa, R. C.; Parton, R. F.; Neys, P. E.; Lardinois, O.; Jacobs, P. A.; Rouxhet, P. G.; J. Molec. Catal. 1996, 110, 141.

12. Alemu, H.; Chandrasvanshi, B. S.; Electroanalysis 1998, 10, 116.

13. Walcarius, A; Electroanalysis 1998, 10, 1217.

14. Perez, E. F.; Kubota, L. T.; Tanaka, A. A.; Oliveira Neto, G.; Electroanalysis 1998, 10, 111.

15. Kubota, L. T.; Gushikem, Y.; Electrochim. Acta 1992, 37, 2480.

16. Denofre, S.; Gushikem, Y.; J. Chem. Soc. Faraday Trans. 1993, 89, 1057.

17. Perez, E. F.; Kubota, L. T.; Tanaka, A. A.; Oliveira Neto, G.; Electrochim. Acta 1998, 43, 1665.

18. Ottaway, J. M.; Bishop; E.(ed); Indicators; Pergamon Press; Oxford; 1972, p. 469.

19. Pessôa, C. A.; Gushikem, Y.; Kubota, L. K.; Gorton, L.; J. Electroanal. Chem. 1997, 431, 23. 